\title{
Überall, Martina
}

\section{Nachhaltigkeitsbildung in der Lehrveranstaltung Ernährungsökologie. Eine Explorationsstudie \\ Haushalt in Bildung \& Forschung 6 (2017) 4, S. 47-62}

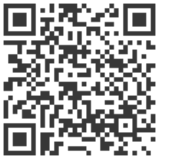

Quellenangabe/ Reference:

Überall, Martina: Nachhaltigkeitsbildung in der Lehrveranstaltung Ernährungsökologie. Eine

Explorationsstudie - In: Haushalt in Bildung \& Forschung 6 (2017) 4, S. 47-62 - URN:

urn:nbn:de:0111-pedocs-191735 - DOI: 10.25656/01:19173

https://nbn-resolving.org/urn:nbn:de:0111-pedocs-191735

https://doi.org/10.25656/01:19173

in Kooperation mit / in cooperation with:

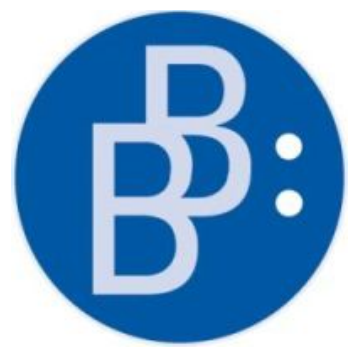

https://www.budrich.de

\section{Nutzungsbedingungen}

Gewährt wird ein nicht exklusives, nicht übertragbares, persönliches und beschränktes Recht auf Nutzung dieses Dokuments. Dieses Dokument ist ausschließlich für den persönlichen, nicht-kommerziellen Gebrauch bestimmt. Die Nutzung stellt keine Übertragung des Eigentumsrechts an diesem Dokument dar und gilt vorbehaltlich der folgenden Einschränkungen: Auf sämtlichen Kopien dieses Dokuments müssen alle Urheberrechtshinweise und sonstigen Hinweise auf gesetzlichen Schutz beibehalten werden. Sie dürfen dieses Dokument nicht in irgendeiner Weise abändern, noch dürfen Sie dieses Dokument für öffentliche oder kommerzielle Zwecke vervielfältigen, öffentlich ausstellen, aufführen, vertreiben oder anderweitig nutzen.

Mit der Verwendung dieses Dokuments erkennen Sie die Nutzungsbedingungen an.

\section{Terms of use}

We grant a non-exclusive, non-transferable, individual and limited right to using this document.

This document is solely intended for your personal, non-commercial use. Use of this document does not include any transfer of property rights and it is conditional to the following limitations: All of the copies of this documents must retain all copyright information and other information regarding legal protection. You are not allowed to alter this document in any way, to copy it for public or commercial purposes, to exhibit the document in public, to perform, distribute or otherwise use the document in public.

By using this particular document, you accept the above-stated conditions of use.

\section{Kontakt / Contact:}

\section{peDOCs}

DIPF | Leibniz-Institut für Bildungsforschung und Bildungsinformation Informationszentrum (IZ) Bildung

E-Mail: pedocs@dipf.de

Internet: www.pedocs.de

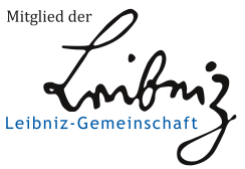


6. Jahrgang Heft 4

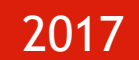$$
4
$$$$
\text { a }
$$$$
>
$$

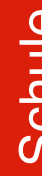

(

(1)

이

1

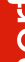

ᄃ

额

(2)

2

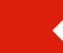

(1)

\section{Bildung in \\ Forschung}

\section{Lernwirksamkeitsmessung II Praxiserfahrungen Ergebnisse}

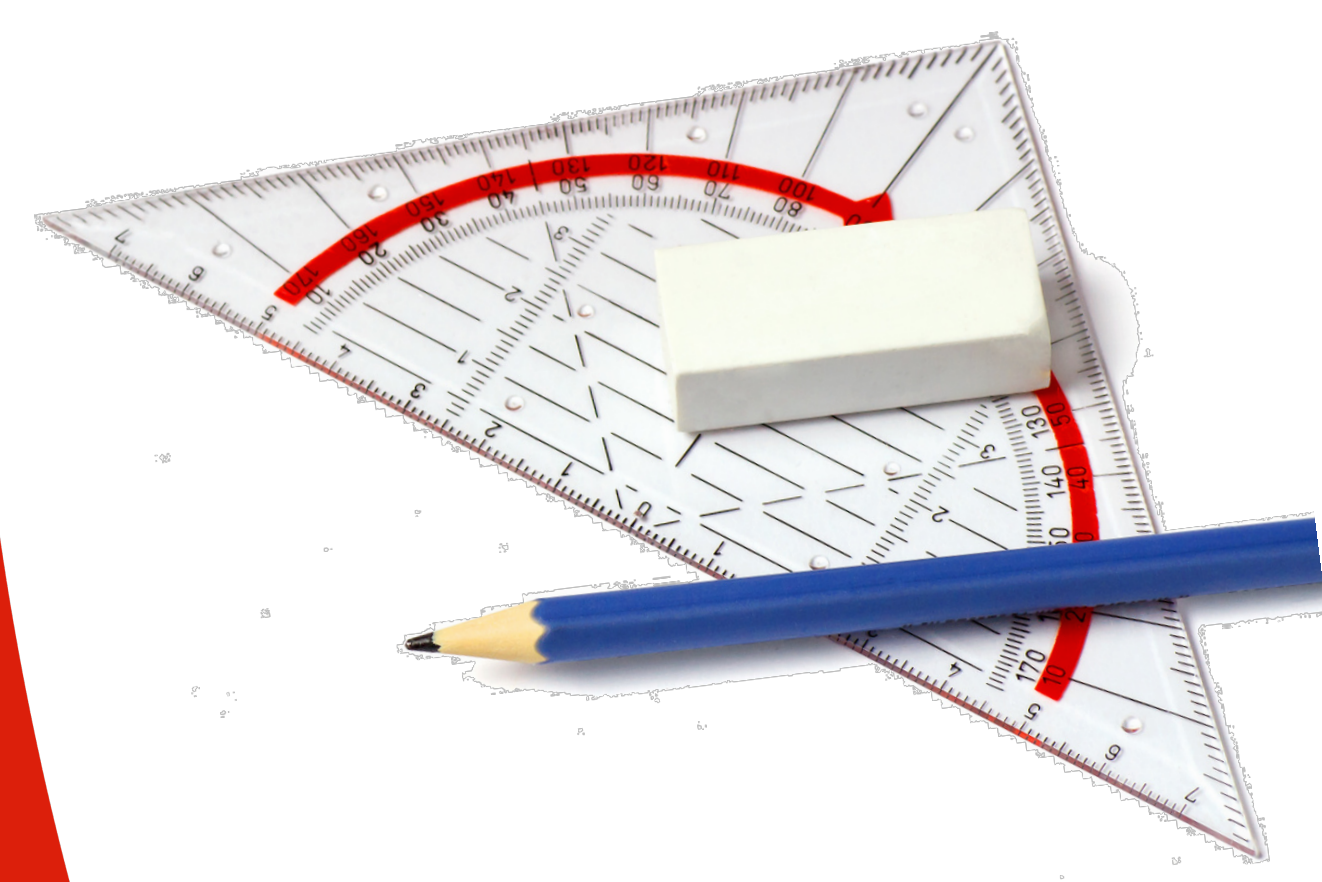

ISSN 2193-8806 


\section{Ursula Buchner}

Editorial.

Gerda Tornieporth, Haido Boudodimos \& Christiane Palmer

Ein Unterrichtsexperiment zum handelnden Lernen.

Susanne Obermoser

„SINNhaftes“ Lernen von abstrakten Begriffen -

sekundäre Pflanzenstoffe.

Gabriela Leitner

Zwischen Skylla und Charybdis: Entwicklung

von moralischen Urteilen im Fachbereich Ernährung.....

Martina Überall

Nachhaltigkeitsbildung in der Lehrveranstaltung Ernährungsökologie:

eine Explorationsstudie

Ursula Buchner, Klara Exner, Lisa Frauenlob, Silvia Meißl \& Lisa Mühlbauer

Einblicke in die Praxis der Lernwirksamkeitsmessung.

Ursula Buchner \& Maria Magdalena Fritz

Fachlich anspruchsvolles Lernen mit Feedback 80

Ursula Buchner

Impulse für kooperative Forschungsprojekte.

Christoph Bohne \& Jana Hinneburg

Verbraucherbildung mit digitalen Medien in der Fort- und Weiterbildung von Berufsschulpersonal.

Call for Papers

HiBiFo Heft 2/2018: Ernährungs- und Verbraucherbildung

in Zeiten von Moden, Mythen, Moral, Medien, Macht 126 


\section{Martina Überall}

\section{Nachhaltigkeitsbildung in der Lehrveranstaltung Ernährungsökologie: eine Explorationsstudie}

Zur zielgruppenorientierten Weiterentwicklung der Lehrveranstaltung Ernährungsökologie wurde ein Forschungs- und Entwicklungsprojekt konzipiert und durchgeführt. Ziel war die empirische Erhebung vom Zuwachs an relevantem, anwendungsorientiertem Fachwissen. Mittels ausgewählter Erhebungsinstrumente, eingesetzt in einer Prä- sowie Posttestung, wurde der Learning Outcome der Lehrveranstaltung analysiert.

Schlüsselwörter: BiNE, Nachhaltige Ernährung, Ernährungsökologie, Lernwirksamkeit

\section{Hintergrund und Forschungsziel}

Bildung und damit auch LehrerInnenbildung spielen eine zentrale Rolle, um Menschen zu befähigen, die Welt zukunftsfähig zu gestalten.

(Steiner \& Rauch, 2013, S. 3)

Dieser Artikel knüpft mit dem durchgeführten Projekt an die Publikation von Überall, Lerchbaumer, Meliss \& Wild (2016) an, wo die Wichtigkeit von Nachhaltigkeit und Ernährung mit Genuss, ganz im Sinne der Lernwirksamkeit, aufgezeigt wurde. Primäre Intention dieses Projektes war das Thema der Nachhaltigen Ernährung ${ }^{1}$ im Rahmen der Lehrveranstaltung Ernährungsökologie in den Lernalltag der ErnährungspädagogInnen zu integrieren und die Effektivität des Unterrichts anhand von Lernstandmessungen zu erheben. Dies geschah im Auftrag, Bildung für Nachhaltige Entwicklung (BiNE) bei den Lehramtsstudierenden fachspezifisch zu verankern und sie dadurch anwendungsorientiert zu befähigen.

Inhaltlich gesehen fokussiert dieses Projekt dabei die im September 2015 von 193 Regierungen dieser Erde beschlossenen globalen Entwicklungsziele. Diese verlangen eine praktikable Strategie, die von informierten Multiplikatorinnen und Multiplikatoren in allen Ebenen unserer Gesellschaft zur Umsetzung gebracht wird. Die insgesamt 17 politischen Zielsetzungen zur Nachhaltigen Entwicklung der Vereinten Nationen (englisch: Sustainable Development Goals, SDGs; Synonyme: „globale Nachhaltigkeitsagenda“, ,2030-Agenda“) umfassen unter anderem:

- „hochwertige Bildung - Inklusive, gerechte und hochwertige Bildung gewährleisten und Möglichkeiten des lebenslangen Lernens für alle fördern." (Nr. 4), 
- „kein Hunger - Den Hunger beenden, Ernährungssicherheit und eine bessere Ernährung erreichen und eine nachhaltige Landwirtschaft fördern.“(Nr. 2),

- „Gesundheit und Wohlergehen - Ein gesundes Leben für alle Menschen jeden Alters gewährleisten und ihr Wohlergehen fördern." (Nr. 3), und

- „nachhaltiger Konsum und Produktion - Für nachhaltige Konsum- und Produktionsmuster sorgen.“(Nr. 12). (BMVIT, 2017)

Exemplarisch aufgezeigt sind hier die vier Ziele, welche für Lehrkräfte in Ernährungspädagogik von größter Relevanz für ihren Wirkungsbereich sind, somit inhaltlicher Fokus facheinschlägiger BiNE und Ausgangspunkt der untersuchten Lehrveranstaltung. Ernährungsbildung ist ein zentrales Handlungsfeld für die „Vermittlung zentraler Kompetenzen für die Lebensgestaltung und insbesondere der Gesundheitsförderung“" (D-A-CH Arbeitsgruppe), sowie eben auch der nachhaltigen Entwicklung. Der Fachbereich Berufsbildung Ernährung spannt diesbezüglich ein potentiell wirksames theoretisches und praktisches Handlungsfeld auf, um einen emanzipatorischen Zugang zur nachhaltigen Entwicklung zu vermitteln (Rieckmann, Fischer \& Richter, 2014, S. 5). Denn zeitgemäße Ernährungsbildung zielt darauf ab, Heranwachsende zu einem selbstbestimmten und verantwortlichen Essverhalten in sozialer und kultureller Eingebundenheit zu befähigen (D-A-CH Arbeitsgruppe). Und auch BiNE generell fungiert als Befähigung zur Selbstbestimmung und dient der Möglichkeit zur gesellschaftlichen Teilhabe. De Haan argumentiert, es geht um die „Eröffnung von Möglichkeiten“ (ebd., 2008, S. 123), daher darf BiNE nicht limitierend oder gar auf ein bestimmtes nachhaltigkeitskonformes Verhalten abzielen. Es geht folglich in der Lehrveranstaltung Ernährungsökologie primär darum, Lerngelegenheiten zu ermöglichen und die Welt aus unterschiedlichen Perspektiven zu erschlieBen.

So wurde diese Explorationsstudie im Rahmen der Lehrveranstaltung Ernährungsökologie zum Thema der Nachhaltigen Ernährung und den Entwicklungszielen 2 und 12 konzipiert.

\subsection{Erschließung Lernwirksamkeit und Nachhaltigkeit}

Zur erfolgreichen BiNE ist „die Entwicklung von fachlichen und persönlichen Kompetenzen der Lehrenden durch Aus- und Weiterbildung [...] ein Schlüssel für den Erfolg [...] - und daher vorrangig zu fördern" (BMFLUW, BMUKK, BMWF, 2008, S. 8). Neben Antworten auf die Fragen: „Was verstehen Studierende im Studiengang Ernährungspädagogik unter Nachhaltiger Ernährung?“" „Auf welche Begriffe und Konzepte greifen sie dabei zurück?“" „Wie wichtig ist ihnen nachhaltiges Essen?“ war es daher Ziel, die Lernwirksamkeit der gewählten Interventionen (Kombination an Unterrichtsmethoden) zu analysieren. 
Betrachtet man Lernen als ,eine quantitative und/oder qualitative Änderung von Wissen und Können, der Fähigkeiten und Fertigkeiten kognitiver, motivationaler volitionaler, aktionaler, emotionaler, sozialer Provenienz beim Lernenden" (Brandl, 2016, S. 4), so ist die Herausforderung der Lernwirksamkeits(er-)forschung, den Lernstand $\mathrm{zu}$ erheben, um zu einem späteren Zeitpunkt eine positive Veränderung (,mehr bzw. besser") nachweisen zu können und diese auch zu dokumentieren und $\mathrm{zu}$ interpretieren.

Im Sinne der Kompetenzorientierung nach Roth (1971), wo eine „Kompetenz als Fähigkeit einer Person, die in bestimmen Situationen sinnvoll zu handeln imstande ist, und damit verschiedene Aufgaben und Lebenssituationen bewältigt" verstanden wird (Dachtler-Freiler, 2016, S. 64), darf diese Erhebung nun selbstverständlich nicht nur rein auf der Wissensebene ansetzen, sondern muss auch die Handlungsfähigkeit betreffender Person analysieren und Veränderungen messen. Insbesondere „Kompetenzen zur eigenen Gesunderhaltung und zum nachhaltigen Konsum können nicht allein auf Wissensvermittlung beruhen, hier spielt auch die motivational-volitionale Dimension (nach Weinert, 2001) eine wichtige Rolle“ (Dachtler-Freiler, 2016, S. $65)$.

Folglich muss der Input (Voraussetzungen und Bedingungen) dem Outcome (Ergebnisse und Wirkungen) gegenübergestellt werden, und eine Prozessorientierung (Throughput) in der Beobachtung scheint sinnvoll. Gleichzeitig stimmt die Autorin dem von Brandl diskutierten „Etikettenschwindel“ zu, wenn er schreibt, dass wo früher Lernziele standen, nun Kompetenzen gelistet werden, die Differenz von Wissen und Kompetenz aber als ein komplexes Gefüge verstanden werden muss (ebd., 2016, S. 4). Ein nachhaltiges oder längerfristiges Endergebnis (Outcome) wurde nicht erhoben, da dieser Status nicht unmittelbar im Anschluss an die Lehrveranstaltung sichtbar wäre, sondern zeitlich gesehen zu einem erheblich späteren Zeitpunkt. Auch kann zwischen Lernfortschritten im formalen Setting des Unterrichts und denen der informellen Lernumgebung der Studierenden nicht differenziert werden. Die Ergebnisse setzen sich aus einer subjektiven Erfassung des Individuums selbst, einer Eigeneinschätzung der eigenen Kompetenzen in einem Lerntagebuch, und einer objektivierten Erfassung mittels Fragebogen zusammen.

Erhoben wurde im Rahmen dieses Projektes nicht nur fachspezifisches Wissen, sondern auch Charakteristika der Dimension Können: „kritisches Denken und Reflexionsorientierung“; „,den Umgang mit Komplexität“; „Fähigkeit zum Problemlösen“; „Partizipation und Verantwortlichkeit“ sowie ,eigenständiges Handeln und Gestalten“ (nach Rauch \& Steiner, 2012, S. 17-18). Weiters wurden Elemente der ,erweiterten Ernährungskompetenz in einer globalisierten (Ess-)Welt" nach Bartsch \& Methfessel (2016) in Betracht gezogen, wie beschrieben in Überall, Lerchbaumer, Meliss \& Wild (2016, S. 80). Ganz im Sinne des österreichischen Modells KOMBiNE (Kompetenzen für Bildung für Nachhaltige Entwicklung), wurde innerhalb des Lehrsettings ein förderliches Lernklima sowie eine adäquate Lernumgebung geschaf- 
fen, um das Anknüpfen an den individuellen Voraussetzungen der einzelnen Lernenden zu fördern (Rauch \& Steiner, 2012, S. 16). Durch die Auswahl und den Einsatz geeigneter Methoden wurden Beteiligungsmöglichkeiten für die Lernenden erwirkt (Rauch, Streissler \& Steiner, 2008). Ein großer Vorteil der Ernährungs-, Konsumenten und Verbraucherbildung ist auch, dass der Lebensweltbezug nicht erst künstlich hergestellt werden muss (Bartsch, 2015, S. 78) und so Aufgaben mit authentischem Lebensweltbezug (nach Meier et al., 2010, S. 89; in Brandl, 2016, S. 16) zur Erhebung verwendet werden konnten.

\subsection{Basis der Explorationsstudie}

In Anknüpfung an Überall, Lerchbaumer, Meliss \& Wild (2016), wo die Wichtigkeit der Verknüpfung von Nachhaltigkeit und Ernährung (mit Genuss, denn Nachhaltigkeit muss schmecken; ebd., 2016) im Sinne der Lernwirksamkeit im Vorfeld aufgezeigt wurde, galt es im Rahmen dieser Explorationsstudie nun tiefergehend zu eruieren, wie das Thema bei jungen Erwachsenen, konkret: Studierenden im 6. Semester des Studienganges der Berufspädagogik Ernährungspädagogik, anschlussfähig gemacht werden kann. Im Zuge dessen wurden die unterschiedlichen Einstellungen und Meinungen der Studierenden, basierend auf dem Fragebogen, welcher dankenswerterweise verwendet werden durfte, einer vorhergehenden Studie und der Veröffentlichung: „Subjektive Theorien von Studierenden zur Nachhaltigen Ernährung“ (Bartsch, 2015), erhoben.

Die primäre Forschungsfrage lautet dabei: Inwiefern trägt der fachliche Fokus auf Ernährungsökologie zur lernwirksamen Kompetenz- und persönlichen Horizonterweiterung hinsichtlich Ernährungs- und Konsumverhalten bei? Zwei inhaltliche Schwerpunkte wurden zur besseren Messbarkeit gesetzt: 1) Spezifische Strategien zur Resteverwertung um Lebensmittelverschwendung zu verringern; 2) Verringerung des Fleischkonsums zur Reduktion des CO2-Ausstoßes.

Dieser Forschungsteil wurde weiters angelehnt an das Schweizer Forschungsund Entwicklungsprojekt von Hertrampf, Städeli \& Bender (2014) und der Veröffentlichung: „Nachhaltige Ernährung - Weiterentwicklung fachwissenschaftlichfachpraktischer Lehrveranstaltungen an der Hochschule“. Es durfte der bereits pilotierte Fragebogen der Schweizer Kolleginnen als Messinstrument zur Posttestung angewandt werden.

\section{Vorgehensweise}

Das Forschungsprojekt wurde im Sinne der Aktionsforschung nach Lawrence Stenhouse und John Elliott konzipiert, welche als umfassende Strategie gilt, um forschendes Lernen und Entwickeln für die eigene berufliche Praxis anzuregen (Soukup-Altrichter \& Altrichter, 2007). 
Fokusgruppe waren insgesamt sieben weibliche Kandidatinnen und ein männlicher Kandidat. Aufgrund der geringen Teilnehmerzahl können keine aussagekräftigen Zusammenhänge zwischen Ergebnis und Geschlecht getroffen werden. Die Studierenden befanden sich zum Erhebungszeitpunkt im letzten Semester ihres Bachelorstudiums und absolvierten alle die Lehrveranstaltung Ernährungsökologie. Zum Vergleich wurden auch alle Studierenden, insgesamt zwölf weibliche Kandidatinnen und zwei männliche Kandidaten, des zweiten Semesters befragt. Die Studierenden waren zum Zeitpunkt der Erhebung alle zwischen 18 und 25 Jahren alt.

$\mathrm{Zu}$ Beginn der Lehrveranstaltung wurde ein Prätest mittels anonymisiertem online Fragebogen 1 und dem österreichischen „ökologischen Fußabdruck“ (BMLFUW, 2017) zur Feststellung des Wissenstandes beziehungsweise der Wissenslücken bei den Studierenden durchgeführt. Fragebogen 1 gliederte sich in offene Fragen (z.B.: „Was verstehen Sie unter nachhaltiger Ernährung?") sowie geschlossenen Fragen (z.B.: „Ist Ihnen persönlich Nachhaltigkeit beim Essen wichtig?“), angelehnt an Bartsch (2015). Es erfolgte eine quantitative sowie eine inhaltsanalytische Auswertung, wie von Bartsch (2015) beschrieben.

Während der Lehrveranstaltung verfassten die Studierenden jeweils am Ende bzw. direkt im Anschluss an die Lehrveranstaltung einen Beitrag in ihrem Lerntagebuch. Dieses diente dazu Sichtstrukturen und Tiefenstrukturen des Unterrichts zu erheben (Heymann, 2015) und auch dazu, den Prozess der Induktion eines Problems beim Lernenden sichtbar zu machen, sowie Fragen nach der kognitiven Aktivierung und der Qualität der inhaltlichen Auseinandersetzung zu beantworten (Brandl, 2016, S. 14).

Abschließend, am Ende des Semesters, wurde als Posttest sowohl der initiale Fragebogen 1 sowie der Fußabdruck noch einmal ausgefüllt und die Studierenden schrieben individuell eine kurze Gesamtreflexion in das Lerntagebuch. Zusätzlich kam in der Posttestung Fragebogen 2 (Hertrampf, Städeli \& Bender, 2014) zum Einsatz. Fragebogen 2 arbeitet mit offenen und geschlossenen Fragen und umfasst 14 Fragekomplexe mit diversen Unterfragen. Er erfasst überwiegend anwendungsorientiertes Fachwissen und bringt die Testperson in konflikthafte Situationen, wo erlernte Fähigkeiten und erworbene Kompetenzen sowie vernetztes Denken in ihren Entscheidungsprozessen unter Beweis gestellt werden können (Hertrampf, Städeli \& Bender, 2014, S. 54). Das Lerntagebuch diente dabei ergänzend der qualitativen Evaluierung, insbesondere um zu erheben, welche Wissenslücken nicht nur theoretisch erschlossen wurden, sondern auch in Handlungsoptionen mündeten.

\section{Ergebnisse und Diskussion}

Subjektive Theorien sind Ausgangslagen des Lernens (Bartsch 2008, 2012; Bartsch \& Methfessel, 2014). Daher war es Ziel im ersten Schritt und im Vorfeld zur Lehrveranstaltung die Einstellungen und Meinungen der Studierenden zur Nachhaltigen 
Ernährung zu erfassen, um anschließend ein zielgruppenorientiertes Gesamtkonzept erstellen zu können.

\subsection{Einstellungsmessung zur Nachhaltigen Ernährung}

Mittels Fragebogen 1 wurden die Auffassungen und Meinungen der Studierenden $(n=8)$ zur Nachhaltigen Ernährung mit der Frage „Was verstehen Sie unter nachhaltiger Ernährung?" erhoben und analysiert und die Antworten den Sieben Grundsätzen einer nachhaltigen Ernährung (nach Koerber, Männle \& Leitzmann, 2012, S. 110) zugeteilt (vgl. Tab. 1). Auch den Fünf Dimensionen der Ernährungsökologie (nach Koerber, 2014, S. 261) wurden die Antworten zugeordnet (vgl. Tab. 2). Die sieben Grundsätze entsprechen dabei konkreten Handlungsempfehlungen zur Umsetzung der fünf Dimensionen im Essalltag. Bei der Auswertung wurde die Zuordnung wie von der Fragebogenerstellerin demonstriert vorgenommen (Bartsch, 2015).

Tab. 1: Studierendenantworten mit Einbezug der Sieben Grundsätze einer nachhaltigen

Ernährung vor und nach der Lehrveranstaltung.

\begin{tabular}{c|c|c}
\hline & \multicolumn{2}{|c}{ Anzahl der Antworten } \\
\hline Grundsätze einer Nachhaltigen Ernährung & Prätest & Posttest \\
\hline 1) Bevorzugung pflanzlicher Lebensmittel & 1 & 1 \\
\hline 2) ökologisch erzeugte Lebensmittel & 5 & 5 \\
\hline 3) regionale und saisonale Erzeugnisse & 3 & 6 \\
\hline 4) Bevorzugung gering verarbeiteter Lebensmittel & 0 & 1 \\
\hline 5) umweltverträglich verpackte Lebensmittel & 0 & 1 \\
\hline 6) fair gehandelte Lebensmittel & 1 & 3 \\
\hline 7) genussvolle und bekömmliche Speisen & 0 & 0 \\
\hline Ergänzend: Future-Generations-Gedanke & 3 & 2 \\
\hline Ergänzend: No-Food-Waste-Gedanke & 1 & 1 \\
\hline Ergänzend: Ökologischer Fußabdruck & 2 & 2 \\
\hline
\end{tabular}

Aus beiden Zuordnungen geht hervor, dass die ökologische Erzeugung von Lebensmitteln im Alltagsverständnis der Studierenden sehr prominent ist. Auch assoziieren sie die Begriffe „regional“ und „saisonal“ mit nachhaltiger Ernährung und der Gedanke, dass wir so leben müssen, dass nachfolgende Generationen noch genügend Ressourcen zur Verfügung haben (betitelt als „Future-Generations-Gedanke“) ist im Verständnis der Studierenden deutlich präsent. Dass ,genussvolle und bekömmliche 
Speisen" auch ein Grundsatz der Nachhaltigen Ernährung ist, begreifen die Studierenden, indem sie über den positiven Einfluss nachhaltiger Lebensmittel auf die Gesundheit schreiben.

Tab. 2: Studierendenantworten mit Einbezug der Fünf Dimensionen einer nachhaltigen

Ernährung vor und nach der Lehrveranstaltung.

\begin{tabular}{c|c|c}
\hline \multicolumn{2}{|c|}{} & \multicolumn{2}{|c}{ Anzahl der Antworten } \\
\hline Dimensionen einer Nachhaltigen Ernährung & Prätest & Posttest \\
\hline A) ökologisch & 6 & 7 \\
\hline B) sozial & 2 & 4 \\
\hline C) ökonomisch & 1 & 1 \\
\hline D) gesundheitlich & 5 & 3 \\
\hline E) kulturell & 1 & 0 \\
\hline
\end{tabular}

Das Konzept der ökologischen Lebensmittelherstellung wird nach der unterrichtlichen Intervention elaborierter auch mit Faktoren wie Transportweg erwähnt (explizite Nennung). Die Wahrnehmung für Qualitätsmerkmale wie „Gütesiegel“ wurde zwischenzeitlich geschärft (explizite Nennung). Bei der offenen Fragestellung werden ,genussvolle und bekömmliche Speisen“ nicht explizit erwähnt, jedoch geht aus den Lerntagebüchern hervor, dass es hier zum Lernzuwachs gekommen ist (nach einer Verkostung im Unterricht), beziehungsweise dieses Konzept zum Zeitpunkt des Posttests präsent im Gedächtnis ist. Die Auswirkungen Nachhaltiger Ernährung auf die Gesundheit wurden mehrmals impliziert. Beispielsweise schreibt ein Student oder eine Studentin: „,Für mich gehört auch zur nachhaltigen Ernährung auf die Qualität der Lebensmittel zu achten!“

Diese Ergebnisse, wenn auch aufgrund der geringen Stichprobe nicht signifikant, zeigen ganz ähnliche Trends, wie sie auch an der PH Karlsruhe erhoben und analysiert wurden (Bartsch, 2015, S. 84).

\subsection{Persönliche Wichtigkeit von Nachhaltiger Ernährung}

Auf die Frage nach der persönlichen Wichtigkeit von Nachhaltiger Ernährung, geben sieben Studierende vor der Lehrveranstaltung „Ja, NE ist mir wichtig“ an und eine Person wählte „keine Meinung“. Die Bereitschaft der Studierenden zur Auseinandersetzung mit dem Thema ist demnach bereits im Vorfeld hoch und Interesse scheint als positive Voraussetzung und Motivator zu bestehen. Im Anschluss an die Lehrveranstaltung, geben nun alle Studierenden „Ja, NE ist mir wichtig“ an. Obwohl während der Lehrveranstaltung ganz besonders auf ein Lernklima geachtet wurde, wel- 
ches freie Meinungsäußerung und persönliche Stellungsnahmen fördert, lässt sich der Einflussfaktor der ,sozialen Erwünschtheit“ hier nicht ausschließen.

\subsection{Subjektive Theorien und eigenes Essverhalten - Lebensweltbezug}

Auch wurden die Studierenden gefragt: „Wie wichtig sind Ihnen folgende Kriterien beim Essen?", wobei sie ein Maximum an 5 Punkten (je mehr desto wichtiger) vergeben konnten. Folgende Mittelwerte und Standardfehler (vgl. Tab. 3) wurden zu den Kriterien vor und nach der Lehrveranstaltung erhoben.

Tab. 3: Studierendenantworten zur persönlichen Wichtigkeit einiger Kriterien beim Essen.

\begin{tabular}{c|c|c}
\hline & \multicolumn{2}{|c}{$\begin{array}{c}\text { Mittelwert der Antworten aus } \\
\text { 5 / Standardfehler (n = 8) }\end{array}$} \\
\hline $\begin{array}{l}\text { Persönliche Wichtigkeit der Kriterien beim Essen } \\
\text { (max. 5 Punkte) }\end{array}$ & Prätest & Posttest \\
\hline a) $\quad$ Fleischreiche Kost & $1,38 / 0,18$ & $1,50 / 0,27$ \\
\hline b) $\quad$ Fair-gehandelte Lebensmittel & $2,38 / 0,32$ & $3,13 / 0,23$ \\
\hline c) $\quad$ Bio-Lebensmittel & $2,75 / 0,25$ & $3,25 / 0,25$ \\
\hline d) $\quad$ Unverpackt/umweltfreundlich verpackte Lm & $3,38 / 0,32$ & $3,63 / 0,18$ \\
\hline e) $\quad$ Lebensmittel aus der Region & $4,13 / 0,13$ & $4,63 / 0,18$ \\
\hline f) $\quad$ Saisonale Lebensmittel & $4,38 / 0,18$ & $4,63 / 0,18$ \\
\hline g) $\quad$ Gesundheitsförderliches Essen & $4,38 / 0,18$ & $4,00 / 0,27$ \\
\hline h) $\quad$ Vermeidung von Lebensmittelverschwen- & $4,50 / 0,27$ & $4,63 / 0,18$ \\
\hline i) $\quad$ Frischkost, d.h. geringe Verarbeitung & $4,75 / 0,16$ & $4,63 / 0,18$ \\
\hline j) $\quad$ Genuss und bekömmliche Kost & $4,75 / 0,16$ & $4,75 / 0,16$ \\
\hline
\end{tabular}

Auffallend ist dabei, dass „Genuss und bekömmliche Kost“ zwar in den Köpfen der Studierenden noch nicht mit Nachhaltiger Ernährung in Zusammenhang steht, aber für diese Zielgruppe einen besonders hohen Stellenwert einnimmt. Dies unterstützt den bereits geäußerten Grundgedanken, dass Genusstraining ein integraler Bestandteil lernwirksamer Lehreinheiten zur Nachhaltigen Ernährung sein muss und „Nachhaltigkeit schmeckt!“" ein erfolgsbringendes Konzept sein kann (Überall, Lerchbaumer, Meliss \& Wild, 2016, S. 17). Auch das Kriterium „Frischkost“ ist der Gruppe besonders wichtig. Dies setzt eine gewisse Zubereitungskompetenz voraussetzt, die 
bei dieser facheinschlägigen Studierendengruppe, verglichen mit anderen Studiengängen, ausgeprägter ist.

Das eigene Essverhalten betreffend wurden die Studierenden nach der Lehrveranstaltung wieder nach der persönlichen Wichtigkeit folgender Kriterien befragt und die Mittelwerte. Während „Genuss und bekömmliche Kost“ immer noch den höchsten Stellenwert erfährt, haben „Bio-Lebensmittel“, „Lebensmittel aus der Region“, „Fair-gehandelte“ sowie „saisonale“ Lebensmittel und „Vermeidung von Lebensmittelverschwendung" nun einen etwas höheren Wert im subjektiven Ermessen der Studierenden erfahren.

\subsection{Der ökologische Fußabdruck}

Der durchschnittliche Gesamtfußabdruck eines in Österreich lebenden Weltbürgers beträgt 5,31 globale Hektar (gha) (BMLFUW, 2017). Ernährung und Essen verursachen den Löwenanteil an diesem Referenzmaß des ökologischen Fußabdruckes, nämlich 1,25 globale Hektar (gha) hier in Österreich und somit mehr als Wohnen ( 0,75 gha), Mobilität (0,78 gha) und Konsum allgemein (1, 25 gha).

Bei den Studierenden lag der Gesamtfußabdruck vor der Lehrveranstaltung zwischen 3,61 und 6,74 globalen Hektar (Einzelwerte: 6,74; 5,39; 4,46; 3,99; 3,88; 3,83; $3,71 ; 3,61)$, berechnet mit dem Tool des Ministeriums für Land- und Forstwirtschaft, Umwelt und Wasserwirtschaft, BMLFUW (2017). Der Mittelwert der Gruppe lag folglich bei 4,45 gha (Standardfehler: 0,39 gha), etwas niedriger als bei einem durchschnittlichen Österreicher oder einer durchschnittlichen Österreicherin. Auch hier wird ein gewisses Bewusstsein dieser Zielgruppe transparent.

Nach Lehrveranstaltungsende, am Ende des Semesters, wurden die gleichen Items abermalig erhoben und nun lagen die Einzelwerte zwischen 3,5 und 5,78 gha $(5,78 ; 4,77 ; 4,51 ; 4,24 ; 4,23 ; 3,69 ; 3 ; 69 ; 3,5)$, mit einem nun etwas niedrigerem Mittelwert von 4,30 gha (Standardfehler: 0,26 gha).

Die Studierenden wurden überdies gebeten, die Teilbereiche ihres Fußabdrucks separat zu vermerken und so konnte ein durchschnittlicher Wert für den Bereich Ernährung von 0,74 gha vor der Lehrveranstaltung berechnet werden und danach lag der Wert bei 0,6 gha. Im Vergleich mit dem durchschnittlichen Wert von 1,25 gha hier in Österreich bringt dies eine hohes Bewusstsein der Probanden zum Ausdruck.

Der ökologische Fußabdruck hat direkte Relevanz und Aussagekraft über den Stand unserer Gesellschaft hinsichtlich SDGs 2 und 12 (,,kein Hunger - durch Ernährungssicherheit, eine bessere Ernährung sowie eine nachhaltige Landwirtschaft"; „,nachhaltiger Konsum und Produktion“). Diese Komplexitäten und Zusammenhänge wurde mit den Studierenden in der Lehrveranstaltung mit dem Lernspiel Globo „Unser kleines Dorf - Die Welt hat 100 Menschen“ (http://www.teamglobo.net/archives/category/unser-kleines-dorf) sowie dem Konzept und Bildungsset „Ein guter Tag hat 100 Punkte“ (https://www.eingutertag.org/de/) erschlossen. Beide Bil- 
dungsmaterialien haben bei den Studierenden großen Anklang gefunden, da verschiedenste Handlungsmöglichkeiten ausprobiert und reflektiert werden.

\subsection{Strategien zur Nachhaltigen Ernährung}

Mit dem Ziel, die Lernwirksamkeit im Hinblick auf die Kompetenz- und persönliche Horizonterweiterung hinsichtlich Ernährungs- und Konsumverhalten der Studierenden zu erheben, wurden neben dem fachlichen Fokus auf Ernährungsökologie zwei konkrete inhaltliche Schwerpunkte zur besseren Messbarkeit gesetzt:

\subsubsection{Resteverwertung und Lebensmittelverschwendung}

Die Studierenden wurden explizit nach ihren spezifischen Strategien zur Resteverwertung um Lebensmittelverschwendung zu verringern befragt und folgende Antworten wurden vor der Lehrveranstaltung gegeben: neben den bekannten Strategien zu Planung und Vorbereitung (z.B. Liste erstellen), Einkauf und Lagerung (z.B. nicht hungrig einzukaufen, Großpackungen nur produktbezogen), Resteverwertung und Konservierungsstrategien (z.B. Resteverwertung durch Verkochen, Tiefkühlen, etc.) und Einsatz der Sinne zur Überprüfung (z.B. Geschmacks- und Geruchsproben), wurden auch spezielle und kreative Ansätze erwähnt, wie zum Beispiel: „Foodsharing Netzwerke nutzen (Apps zur Lokalisation)“, „Party bzw. Mädelsabend um Reste zu verwerten“ oder „Mindesthaltbarkeitsdatum hinterfragen“.

Auf die abermalige Frage nach ihren Strategien um Lebensmittelverschwendung zu vermeiden, wurden nach der Lehrveranstaltung eine Vielzahl an bekannten Taktiken und auch folgende effiziente Ansätze beigetragen: ,aus den Lebensmitteln, die im Kühlschrank vorhanden sind, ein geeignetes Gericht herstellen“, „Food-sharing mit Freunden/Bekannten oder diese spontan einladen“, „Gerichte für die Woche planen“, „neue Rezepte anhand der übrigen Lebensmittel ausarbeiten“, „genaues Berechnen der Mengen für die Anzahl der Personen“ sowie „Bildung und Aufklärung“. Daraus geht der Wunsch nach aktiver Beteiligung hervor, und die Studierenden nennen mannigfaltige Gestaltungsmöglichkeiten zu den Rahmenbedingungen des Handelns.

Durch Fragebogen 2 wurde zusätzlich Fachwissen zum Verderb, unabhängig vom Mindesthaltbarkeitsdatum von Lebensmitteln, erhoben und auch hier ging ein grundlegend hohes Kompetenzniveau der Studierenden hervor, da sie zum Beispiel alle mit schimmligem Brot oder Joghurt mit Flüssigkeit oben auf umzugehen wussten. Auch, dass Schnellkochtöpfe weniger Energie benötigen als herkömmliche Pfannen war ihnen bekannt, sowie weitere Strategien zur Energieeffizienz als Teil der Nachhaltigen Ernährung. 


\section{Nachhaltigkeit schmeckt! - Teil 2 |}

\subsubsection{Fleischarme und fleischlose Kost}

Auf die Frage: „Wie häufig konsumieren Sie derzeit Fleisch oder Fleischprodukte pro Woche?“, antwortete eine Person „weniger als wöchentlich“, vier Personen mit „1-2 Mal“, eine Person mit „2-3 Mal“ und zwei Personen mit „3-4 Mal“. Alle befragten Personen achteten beim Kauf von Fleisch auf die Qualität. Diese Angaben wurden vor der Lehrveranstaltung gemacht.

Als Antwort auf dieselbe Frage nach ihrem wöchentlichen Fleischkonsum nach der Lehrveranstaltung gaben zwei Personen an „weniger als wöchentlich“, vier Personen „1-2 Mal“, eine Person „2-3 Mal“" und eine Person ,3-4 Mal“. Es gab nun also eine Verlagerung, welche insgesamt einen niedrigeren Fleischkonsum zum Ausdruck bringt, wobei die Aussagekraft bei dieser kleinen Fokusgruppe nicht als signifikant anzusehen ist.

Genauer hinterfragt wurde der Fleischkonsum im Zusammenhang mit Nachhaltiger Ernährung mit Fragebogen 2, der nach einer Entscheidung zwischen „trifft zu“ bzw. „trifft nicht zu“, zum Beispiel zu folgender Aussage befragte: „Fleisch ist für eine gesunde Ernährungsweise unabdingbar und sollte regelmäßig in den Ernährungsplan integriert werden.“. Diese Frage beantworteten alle Kandidatinnen mit „trifft nicht zu“. Die Studierenden waren sich einig, dass „durch die Reduktion des Fleischkonsums die individuelle Treibhausgasemission reduziert werden kann", und auch dabei, dass „regionale Produkte (z.B. Rindfleisch) globalen Produkten stets vorzuziehen sind“. Zwei Personen fanden, dass die Aussage: „Fleischverzehr der Industrienationen hat Auswirkungen auf die Armut der Menschen in den Entwicklungsländern" nicht zutrifft, wobei sechs Studierende der Meinung waren, dass dies zutrifft. Wissenserweiterung erwies die Gruppe bei der Einschätzung, ob ,durch eine vegetarische Lebensmittelauswahl der persönliche Wasserverbrauch verringert wird“" und inwiefern „Rinderzucht die Ökologie stärker beeinträchtigt als Geflügelzucht“. Ihre potentielle Handlungskompetenz, konkretes prozedurales Wissen für den Theorie-Praxis-Transfer, wurde in der anwendungsorientierten Frage: „Welche fleischlosen Gerichte sind Ihnen bereits bekannt? Nennen Sie maximal fünf Gerichte.“, sowie der Folgefrage: „Welche der in der vorhergehenden Frage genannten fleischlosen Gerichte haben Sie im Ernährungsunterricht bereits zubereitet?" erhoben.

Auch nach der Bekanntheit einiger Qualitäts- und Biolabels wurde gefragt und die Gruppe erwies bei Demeter, BioAustria und Bio vom Berg, allen mit Relevanz für den Fleischkauf, hohe Kompetenz in der Beschreibung derer Kriterien (nur das FairTrade-Zeichen wurde noch genauer charakterisiert).

Wird auf Fleisch verzichtet, bzw. der Fleischkonsum verringert, so muss ernährungsphysiologisch die Deckung des Eiweißbedarfs sichergestellt werden. Folglich mussten die Studierenden ihre Wissenskompetenz unter Beweis stellen, indem sie gefragt wurden, Lebensmittel in ihrem Eiweißgehalt einzuschätzen. Auch hierbei schnitt die Gruppe kompetent ab. 


\subsection{Lernwirksame Elemente der Lehrveranstaltung?}

Das Konzept der gesamten Lehrveranstaltung im Detail vorzustellen und die genaue Vorgehensweise zu erläutern, würde den Rahmen der Publikation überschreiten, daher wird hier nur kurz auf die Themenbereiche Resteverwertung und Lebensmittelverschwendung und Fleischarme und fleischlose Kost eingegangen und es werden vorerst nur einige wenige Ergebnisse aus dem Lerntagebuch erwähnt.

\subsubsection{Resteverwertung und Lebensmittelverschwendung}

Hinsichtlich Ressourcennutzung wurde vorab die fachwissenschaftliche Theorie (Wertschöpfungskette (z.B. vom Korn zum Brot), No-Food-Waste-Konzepte, virtuelles Wasser, u.v.m.) anwendungsorientiert erarbeitet. Allgemeine Umsetzungsstrategien wurden diskutiert und analysiert (z.B. „Lebensmittel sind kostbar“ Initiative Österreich, „Zu gut für die Tonne“ Initiative Deutschland plus App, Food-Sharing Plattformen, Food-Coops, etc.). Abschließend wurde eine genussorientierte (denn: Nachhaltigkeit schmeckt!) praktische Einheit mit einem lokalen Verein, dem feldverein, initiiert, dessen Zielsetzung die Nutzung von Ungenutztem ist und die ihre Produkte nach dem Pay-as-you-wish-Konzept vertreiben (feld-verein, 2017). Zur Vertiefung wurde die dort angesiedelte Food-Coop unter die Lupe genommen und genussvolle Resteverwertung praktiziert. $\mathrm{Zu}$ ihren Erkenntnissen schreiben die Studierenden folgendes: „Nur weil ein Kohlrabi zu groß ist, oder eine Karotte eine braune Stelle aufweist werden sie weggeworfen? Das ist wirklich traurig, aber man braucht ja nur im Supermarkt schauen, die Konsumenten erwarten optisch einwandfreies Gemüse. [...] Aber auch hier darf ich mich nicht ausnehmen, man nimmt nicht bewusst das Gemüse, das am bräunlichsten ist“, „[...] Lebensmittelabfälle können entlang der ganzen Wertschöpfungskette vermieden werden. Wir alle können bereits mit kleinen Maßnahmen Großes bewirken“, „Diese Initiativen sind wichtig, damit die Menschen wieder mehr Verständnis für Lebensmittelwertschätzung bekommen“, „Das Konzept des Vereins überzeugt“, „Eine tolle Alternative um zu guten Produkten zu kommen“, „Superspannend ein alternatives Projekt zum konventionellen Handel hautnah zu erleben“, „Gezielte Förderung der Wertschätzung von Lebensmitteln“, „Mir wurde erst so richtig bewusst in welcher Wegwerfgesellschaft wir leben und dass man mit wenigen Lebensmitteln gesunde und schmackhafte Gerichte zubereiten kann“, und „Ich möchte mit Schülerinnen und Schülern ebenso Projekte dieser Art umsetzen".

Wurde eingangs bereits der emanzipatorische Ansatz erwähnt, so wird durch diese Aussage hier eine gewisse Emotionalisierung evident (z.B.: „Es ist wirklich traurig."), die mehr dem ,instrumental approach“ nach Wals (2011) zugeschrieben wird. Unter Bezug auf Ansätze der Umweltpsychologie wurden, wie es scheint, beim feldverein Anreize gegeben, um ein bestimmtes, vermeintlich nachhaltiges Verhalten attraktiv zu machen (Rieckmann, Fischer \& Richter, 2014, S. 4). Die Lehrveranstal- 
tungsleiterin dagegen hat in ihrem Unterricht bewusst vermieden, Stilmittel des moralischen Lernens einzusetzen und einen reflexiven Ansatz gewählt. Denn beim „emancipatory approach“ nach Wals (2011) geht es nicht darum, Denk- oder Verhaltensweisen vorzugeben, sondern Individuen in die Lage zu versetzt, eigenständig über Fragen einer nachhaltigen Entwicklung nachzudenken und ihre eigenen Antworten zu finden (Rieckmann, Fischer \& Richter, 2014, S. 4). Hinsichtlich konnten die Tiefenstrukturen etwas erschlossen werden.

\subsubsection{Fleischarme und fleischlose Kost}

Den Fleischkonsum ins Zentrum rückend, wurde ebenso vorab die fachwissenschaftliche Basis anhand globaler Zusammenhänge erarbeitet (z.B. kleinstrukturierte vs. industrialisierter Landwirtschaft, Futtermitteleinsatz und -herkunft). Auch diverse Initiativen in diesem Themenbereich wurden erläutert und diskutiert (z.B. Vegucation). Abschließend luden wir den Geschäftsführer einer lokalen bäuerlichen Genossenschaft mit eigener Bio-Marke (,Bio vom Berg“) zur Diskussion ein und besuchten einen Demeter-Hof um den lokalen Gegebenheiten praktisch nachzugehen. Die Studierenden äußerten sich im Anschluss an die Lehrveranstaltung folgendermaßen: „Wir wissen, dass für Fleisch sehr viel Wasser benötigt wird, ich denke aber nicht, dass SchülerInnen das so bewusst ist. [...] Es sollte jedem/jeder bewusst sein, wieviel Wasser für die Produktion von Fleisch benötigt wird.“, dieser Aspekt wurde gleich mehrmals betont, „Ich finde es nicht richtig, dass es vegane Wurstprodukte gibt, denn diese sind nicht zwingend gesünder für den Menschen oder für die Umwelt (viele Prozessschritte!)“, „Bei der Vermarktung wird sehr auf Emotionen und landwirtschaftliche Idylle (in Bildern) gebaut. Inwieweit diese Idylle der Wahrheit entspricht, ist fraglich“, „Besonders spannend ist beim Fleischkonsum das Konzept der „Food-Printler. Diese Personen essen hauptsächlich Fleisch von Wild- oder Almtieren. Es ist von größter Bedeutung Fleisch bzw. tierische Lebensmittel bewusst einzukaufen (heimisch, biologisch, Direktvermarktung). Ich möchte mir dieses Vorhaben stets vor Augen halten.“

Diese Thematik eignete sich ideal, um im Anschluss über Emotionalisierung und Wertevorstellungen im Zusammenhang mit nachhaltiger Entwicklung zu diskutieren, was anhand der Einträge in den Lerntagebüchern gezielt aufgegriffen werden konnte. Die Studierenden haben explizit zum Ausdruck gebracht, dass lösungsorientierte Initiativen sehr positiv wirken und „,noch eine Chance für die Weiterentwicklung der Welt besteht.“ Dies war ein Anknüpfungspunkt, um in der nächsten Einheit den lateinamerikanischen Diskurs zum Buen Vivir (Gutes Leben) und zu den Rechten der Natur in der Wertedebatte aufzurollen und damit die eurozentrische Denkweise zu erweitert und kritisch zu reflektieren. 


\section{Resümee \& Ausblick}

Die erhobenen Einstellungen und Meinungen (Ansätze subjektiver Theorien) im Vorfeld zur Lehrveranstaltung lieferten nützliche Hinweise auf den Lernstand der Studierenden, obgleich sie wenig über die in dieser Logik des Denkens enthaltene Wissenskonstruktion aussagen. So gelang es, das Konzept zur Ernährungsökologie begründet und vor allem zielgruppenorientiert weiterzuentwickeln. Die Vorgehensweise (Erhebung im Vorfeld und Lerntagebucheinträge im Anschluss an jede Einheit und Abschlusserhebung) hat sich als zielführend erwiesen, da zeitnah auf die Einträge reagiert werden konnte. Insbesondere in einem Themenfeld wie der Nachhaltigen Entwicklung und Ernährung sind die Ansätze häufig nicht wertfrei. Darüber hinaus decken sich die individuellen Interessen mehrfach nicht mit den Anforderungen an die Gesellschaft, sowie allgemeinen ethischen Ansprüchen zum Gemeinwohl. So scheint es sinnvoll, Lernbarrieren, basierend auf diesen subjektiven Theorien, im Vorfeld zu eruieren und dann gezielt aufzugreifen. Zukünftig soll insbesondere diese Lehrveranstaltung, mit so großem Potential für BiNE, einer noch stärker forschungsgeleiteten Ausrichtung unterliegen. Dabei wird auch eine enge Verknüpfung mit dem fachpraktischen Teil angestrebt. Als Wahlmodul wird künftig zum Beispiel „Vegan/vegetarischer Kochprofi“" (Vegucation) angeboten. Auch die Kochprüfung der Studierenden im Fachbereich Ernährung soll entsprechend umstrukturiert werden, zum Beispiel mit einem regionalen und saisonalen Warenkorb, den die Studierenden „restlos“, zeitgemäß und den ernährungsökologischen Grundsätzen entsprechend zu einem genussbringenden Menü verarbeiten. Diese Explorationsstudie liefert wertvolle erste Ansätze und nicht zuletzt Anregungen, um den Lernprozess zukünftig noch konkreter auszuloten, wobei die gewählten Forschungsmethoden sich bereits in dieser Kombination als hilfreich erwiesen haben. Künftig soll die Ebene der Tiefenstrukturen auch gezielter erhoben werden, nicht zuletzt um die Kluft zwischen Wissen und Handeln, eventuellen Barrieren der Selbstbefähigung, zu erschließen.

\section{Anmerkungen}

1 Die fachwissenschaftliche Basis der Lehrveranstaltung bildeten die Fünf Dimensionen einer nachhaltigen Ernährung (Koerber, 2014, S. 261). Dieses Modell basiert wiederum auf dem Drei-Säulen-Modell der Nachhaltigkeit, welches die ökologische, die ökonomische sowie die soziale Dimension berücksichtigt und noch zusätzlich Gesundheit und Kultur inkorporiert (Koerber, Männle \& Leitzmann, 2012; Koerber, 2014, S. 261). Die Autoren haben dazu Handlungsempfehlungen (Grundsätze einer Nachhaltigen Ernährung) zur Umsetzung im Essalltag erarbeitet (Koerber, 2014, S. 263). Das Gießener Vollwertmodell dieses Ansatzes ist wohlbekannt und auch die Wertschöpfungskette spielt eine maßgebende Rolle mit Be- 
tonung auf ökologischer Produktion und Transport sowie fairem Handel. (Für eine ausführlichere Definition des unterrichteten Konzeptes siehe Überall, Lerchbaumer, Meliss \& Wild, 2016).

\section{Literatur}

Bartsch, S. (2008). Subjektorientierung in der Ernährungs- und Gesundheitsbildung. Ernährung - Wissenschaft und Praxis, 2, 100-106.

Bartsch, S. (2012). Subjektorientierung. Ein Beitrag zur kompetenzorientierten Aufgabengestaltung in der Verbraucherbildung. Haushalt in Bildung \& Forschung, 1(3), 52-64.

Bartsch, S. (2015). Subjektive Theorien von Studierenden zur Nachhaltigen Ernährung. Explorationsstudie. Haushalt in Bildung \& Forschung, 4(4), 78-92. http://dx.doi.org/10.3224/hibifo.v4i4.21295

Bartsch, S. \& Methfessel, B. (2014). „Der subjektive Faktor“. Bildung in einem lebensweltorientierten Fach. Haushalt in Bildung \& Forschung, 3(3), 3-32. https://doi.org/10.3224/hibifo.v3i3.16665

Bartsch, S. \& Methfessel, B. (2016). Ernährungskompetenz in einer globalisierten (Ess-)Welt. Herausforderungen und Erfordernisse. Ernährung im Fokus, 16(3-4), 68-73.

BMFLUW, BMUKK, BMWF (2008). Österreichische Strategie zur Bildung für Nachhaltige Entwicklung. Wien: Eigenvervielfältigung.

BMLFUW, Bundesministerium für Land- und Forstwirtschaft, Umwelt und Wasserwirtschaft. (2017). Mein Fußabdruck. http://www.mein-fussabdruck.at

BMVIT - Bundesministerium für Verkehr, Innovation und Technologie. (2017). Agenda 2030 für nachhaltige Entwicklung.

https://www.bmvit.gv.at/ministerium/agenda2030

Brandl, W. (2016). Lernstand erheben, Lernwirksamkeit feststellen, Lernleistung beurteilen - eine Skizze des Bezugssystems. Haushalt in Bildung \& Forschung, 5(4), 3-24. https://doi.org/10.3224/hibifo.v5i4.25691

D-A-CH Arbeitsgruppe. www.evb-online.de/evb.php

Dachtler-Freiler, I. (2016). Neuer Lehrplan - und was nun? Basisüberlegungen zur Lernwirksamkeit des neuen Lehrplans. Haushalt in Bildung \& Forschung, 5(4), 61-72. https://doi.org/10.3224/hibifo.v5i4.25695

de Haan, G. (2008). Gestaltungskompetenz als Kompetenzkonzept der Bildung für nachhaltige Entwicklung, In I. Bormann \& G. de Haan (Hrsg.): Kompetenzen der Bildung für nachhaltige Entwicklung. Operationalisierung, Messung, Rahmenbedingungen, Befunde (S. 23-43). Wiesbaden: VS Verlag.

feld-verein. (2017). http://feld-verein.at

Felten, E. \& Stern, E. (2012). Lernwirksam unterrichten. Im Schulalltag von der Lernforschung profitieren. Berlin: Cornelsen Scriptor. 
Hertrampf, A., Städeli, R. \& Bender, U. (2014). Nachhaltige Ernährung - Weiterentwicklung fachwissenschaftlich-fachpraktischer Lehrveranstaltungen an der Hochschule. Haushalt in Bildung \& Forschung, 3(2), 48-59. https://doi.org/10.3224/hibifo.v3i2.16312

Koerber, v. K. (2014). Fünf Dimensionen der Nachhaltigen Ernährung und weiterentwickelte Grundsätze. Ein Update. Ernährung im Fokus, 14(5-6), 260-266.

Koerber, v. K., Männle, T. \& Leitzmann, C. (2012). Vollwert-Ernährung - Konzeption einer zeitgemäßen und nachhaltigen Ernährung (11. Aufl.). Stuttgart: Haug Verlag.

Kunter, M. \& Klusmann, U. (2010). Kompetenzmessung bei Lehrkräften - Methodische Herausforderungen. Unterrichtswissenschaft, 38(1), 86-86.

Rauch, F., Streissler, A. \& Steiner, R. (2008). Kompetenzen für Bildung für Nachhaltige Entwicklung (KOM-BiNE). Konzepte und Anregungen für die Praxis. Wien: BMUKK.

Rauch, F. \& Steiner, R. (2012). Welche Kompetenzen braucht Bildung für Nachhaltige Entwicklung? Haushalt in Bildung \& Forschung, 1(1), 13-24.

Rieckmann, M., Fischer, D. \& Richter, S. (2014). Nachhaltige Ernährung im Wertediskurs - Beiträge einer Hochschulbildung für nachhaltige Entwicklung. In: Schank, C., Vorbohle, K., Quandt, J. H. (Hrsg.): Perspektive Nahrungsmittelethik (S. 29-58). München, Mering: Rainer Hampp Verlag.

Steiner, R. \& Rauch, F. (2013). Grundsatzpapier zur Bildung für Nachhaltige Entwicklung in der PädagogInnenbildung Neu. Erstellt im Auftrag des Bundesministeriums für Wissenschaft und Forschung.

Soukup-Altrichter, K. \& Altrichter, H. (2007). Einleitung. Schulentwicklung durch Schulprofilierung? Ziele und Anlage der Untersuchung. In: K. SoukupAltrichter et al.: Was verändert sich durch Schulprofilierung? 45: 13-24.

Überall, M., Lerchbaumer, M., Meliss, C. \& Wild, B. (2016). Nachhaltigkeit schmeckt! Haushalt in Bildung \& Forschung, 5(4), 73-92.

https://doi.org/10.3224/hibifo.v5i4.25696

\section{Verfasserin}

Mag. ${ }^{a}$ Martina Überall, $\mathrm{PhD}$

Pädagogische Hochschule Tirol

Institut für Berufspädagogik, Fachbereich Ernährung \& Gesundheit

Pastorstraße 7

A-6010 Innsbruck

E-Mail: martina.ueberall@ph-tirol.ac.at

Internet: https://ph-tirol.ac.at 